\title{
PLAIDOYER POUR DES NON-ÉVÉNEMENTS: UNE RÉFLEXION SUR LE CENTRE D'ART WAZA
}

\author{
Patrick Mudekereza \\ Waza, Centre d'art de Lubumbashi, République Démocratique du Congo
}

Lorsque je parle du travail du centre d'art Waza en dehors du Congo, vient souvent une série de questions auxquelles je peine à répondre : quel moment est le plus propice pour venir vous rendre visite? Avez-vous un festival ou quelque chose de la sorte? La réponse induite dans la deuxième partie porte à croire que l'organisation d'un événement crée un moment propice pour découvrir une ville et le travail d'une organisation. S'il est vrai que la plupart des biennales et des festivals d'art public mettent un point d'honneur à travailler leur ancrage dans la société, il est tout aussi vrai que la manière dont cette offre est «emballée» pour les visiteurs étrangers crée une distance, une sorte de bulle, qui leur impose une lecture "mondialisée» du contexte plutôt qu'une rencontre profonde et sincère avec ses acteurs. Si le centre d'art a été dans son ancienne forme et sous notre ancienne appellation, l'hôte de la deuxième et de la troisième édition de la Biennale de Lubumbashi en 2010 et 20I3, sa quête de pertinence s'est tournée depuis vers d'autres préoccupations.

La réponse que j'ai envie de donner est celle de faire un plaidoyer pour un non-événement. Pas un événement qui part en vrille, qui ne répond pas aux attentes du public et de la presse, mais un non-événement minutieusement préparé en tant que tel, qui pose les bases d'une rencontre, ose être flexible, se crée avec ceux qui le vivent et se prolonge dans leur quotidien.

Le travail de Waza se trouve à l'intersection entre la création artistique, la recherche et les dynamiques communautaires. Il pose de nouvelles questions à chaque composante. Le défi pour un centre d'art est celui de confronter le processus de la création artistique à l'écosystème qui lui donne naissance, sans tomber dans la tentation de nier ses propres contingences liées au monde de l'art. Il s'agit alors d'équilibrer, comme le ferait un DJ devant une piste de danse, les différentes pulsions comme autant de sonorités qui donnent au public un rôle plus actif dans l'expérience de l'exposition.

Les derniers mois, l'équipe du centre d'art Waza, assistée par la muséologue Sari Middernacht, les artistes et opérateurs culturels partenaires, ainsi que des organisations amies comme le centre d'innovation de Lubumbashi avons réfléchi au sens qu'on pouvait 


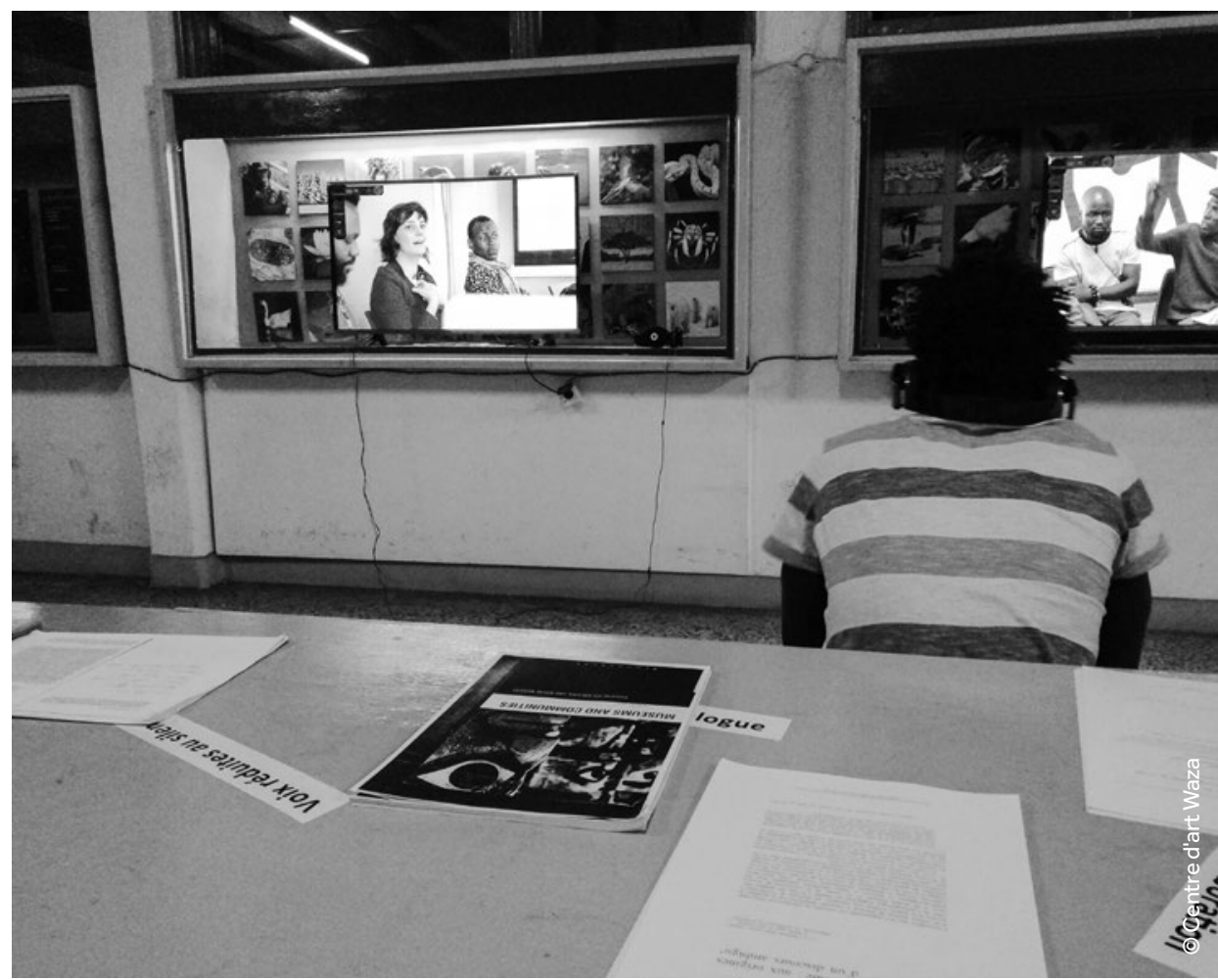

Figure 1: Exposition "Disolo, convers(at)ions avec les collections du musée", Musée National de Lubumbashi, 15 octobre 2018.

donner à un programme qui s'inspire de cette démarche. Entre collecte d'histoires et design thinking, nous avons conçu un nouveau programme en s'inspirant de nos actions passées.

L'expérience de Revolution Room, programme mené en collaboration avec Visual Art Network South Africa de 2013 à 2016 a permis de tester une méthodologie de travail qui part de l'expérience des habitants en partageant l'autorité curatoriale avec eux dans le choix des objets, le sens qui leur est donné. L'exposition Waza chumba Wazi qui a investi pendant un mois une maison du quartier Gécamines a été un élément important du programme à Lubumbashi. D'autres expériences de travail avec les communautés de créations artistiques, de recherche et de présentations publiques ont été réalisées à Fungurume, à Moba pour la partie congolaise et à Cosmo City, un nouveau quartier de Johannesburg pour le volet sud-africain. Plusieurs projets de recherches de création ont été réalisés dans chaque ville comme le projet Misambwa, les ancêtres cosmopolites du sculpteur Agxon, devenu chef coutumier dans un village Tabwa au bord du Lac Tanganyika. Une publication retrace les réflexions critiques et les résultats du projet. Cette publication et le projet ont reçu le prix d'excellence d'African Architecture Award, dans la catégorie 
Critical dialogue en septembre 2017 au Cap, en Afrique du Sud.

Pour Waza, ce projet a participé à une véritable transformation de notre position. Cette transformation a donné lieu à une forme d'hybridation qui rejoint l'idée de Charles Esches d'un espace qui est en partie un centre communautaire, en partie un laboratoire et en partie une académie en réduisant les besoins d'une fonction de salle d'exposition. Dans les activités des dernières années, l'accent a été mis sur le travail collectif: les bédéistes du collectif Les Mines Lushoises, fondé par Daniel Sixte Kakinda, les beatmakers de Mite Empire autour de DJ Spilulu, ou le mouvement de danse contemporaine autour du chorégraphe Dorine Mokha, ont façonné une ligne de programmation et un principe de fonctionnement basés sur l'idée de réimaginer le travail salarié, par des dynamiques collaboratives. Nous avons appelé ce principe de fonctionnement Kazi 2.0, en alliant le concept de Kazi (travail en swahili, qui reprend aussi une certaine idée du monde moderne) à l'idée de l'interactivité du web 2.0 pour contrer les logiques paternalistes des entreprises minières publiques de notre région.

Si cette ouverture a permis de mieux ancrer nos actions en faveur des jeunes artistes lushois, deux éléments ont manqué dans cette configuration : le rythme qui permet de se fixer des étapes pour faire le point de ce qu'on a appris ensemble et une programmation publique qui présente de manière cohérente des artistes locaux et des travaux réalisés par nos partenaires internationaux (artistes ou organisations). Pour ce faire, Waza a mis sur pied un cycle de deux ans qui alterne d'une année laboratoire, Kazi 2.o, revue comme un cadre de partage de connaissance, suivie d'une année de médiation avec le public, Mitaani Moments.

Depuis août 20I8, et pour les 12 mois à venir, Waza s'engage donc dans une série de moments ouverts au public, à Lubumbashi ou ailleurs. Mitaani signifie «dans les villes » ou «dans les quartiers » exprime la volonté de sortir d'une attente d'un lieu culturel formel pour expérimenter d'autres formes de rencontre avec le public, d'autres expériences artistiques. Ces expériences sont des "moments" pour lesquels l'accent est davantage sur le contenu plutôt que sur la visibilité, sur la nature de l'expérience plutôt que sur le nombre de participants. Ces moments se veulent un marqueur interne qui parle aux gens plus qu'un surgissement externe qui les éblouis. Ils se déroulent sous le thème des convers(at)ions urbaines, pour exprimer le potentiel de transformation (conversion) contenu dans un dialogue franc et équitable (conversation) dans l'espace urbain.

Ces conversations se développent sur 4 séquences trimestrielles: archive sur la culture matérielle contenu dans les musées, class sur l'éducation artistique, digital sur le numérique comme outil de diffusion de l'art et enfin mapping sur les cartographies des espaces physiques ou imaginaires. Ces séquences qui se prolongent les unes dans les autres sont des dispositifs qui testent des méthodes d'interactions, des outils de travail et produisent des idées dans la rencontre avec le public. C'est de cette rencontre que se définiront les projets artistiques ou de recherche qui feront l'objet de l'année Kazi 2.o.

La séquence archive a consisté en une série d'activités à Lubumbashi et à Kinshasa en parallèle du cours de Curating Exhibition du département d'histoire de l'art de Wits School of Arts (université de Witwatersrand de Johannesburg). Au Musée National de 
Lubumbashi, l'exposition Disolo, convers(at)ion avec les collections du musée a été organisée du I3 au 20 octobre 20I8, et a présenté les œuvres de Rita Mukebo, Gulda El Magambo, Paul Malaba, Agxon, Hilaire Balu Kuyangiko et Denise Maheho. Cette exposition placée dans le parcours de visite du musée en prolongement de la salle d'ethnographie, questionnait les savoirs sur les objets des collections d'art classique africain conservé au Congo et en Afrique du Sud en intégrant d'une part les interprétations des artistes d'aujourd'hui sur leur sens, et d'autre part en s'engageant à détrôner les savoirs ethnographiques par les réinterprétations des communautés sources. Une salle de documentation, conçue comme une photocopithèque et non comme bibliothèque, rassemblait des photocopies d'articles sur lesquels les annotations, les ratures ou simplement la copie des documents étaient encouragés. Cinq moniteurs présentaient des discussions avec les "papas» Kanteng, Nshimba et Kapenda qui parlaient de leur expérience dans les cultures ruund, luba et tshokwe, les commissaires sud-africains posait le problème de l'interprétation de leur collection d'art congolais qui est conservé en diaspora alors que Philippe Mikobi et Henry Bundjoko expliquaient comment ils naviguent comme anthropologues et historiens de l'art entre les savoirs vivants de leur culture kuba et leele et les interprétations parfois erronées de leurs collègues occidentaux. La salle de projection a aussi servi de salle de discussion, avec la conférence du vernissage qui rassemblait Nontobeko Ntombela (Wits School of Arts), Sarah Van Beurden (Ohio State University) et Philipe Mikobi (Musée National de Lubumbashi). Les débats ont été prolongés au cours d'un atelier sur les perspectives congolaises, sur la restitution des biens culturels et sur la transformation des pratiques muséales en Afrique, organisé par le Centre d'art Waza, à la demande de Goethe Institut Kinshasa.

Les derniers mois, l'équipe du centre d'art Waza, en collaboration avec le projet Another Roadmap for Arts Education, alimentera la séquence class de Mitaani Moments avec les travaux de la commissaire Andrea Thal, de la photographe Rana El Nemr (toutes les deux sont membres du Contemporary Image Collective du Caire) et de l'artiste Christian Nyampeta (Rwanda/Pays-Bas). Les recherches sur l'éducation artistique alternative et les projets qui relatent l'histoire des productions de pensées radicales dans plusieurs contextes en Afrique, en Amérique latine et en Europe seront présentées à des étudiants en pédagogie de Lubumbashi.

Qu'est-ce que ces projets en cours disent de la quête de pertinence du Centre d'art Waza? Ils expriment d'abord une volonté de voir la création artistique dans un spectre plus large, où les questions des savoirs, de justice sociale et de réappropriation de l'imaginaire ne sont plus de simples corolaires, mais font partie intégrante du travail du centre d'art. Ils s'inscrivent ensuite dans une volonté d'adéquation entre les ressources disponibles dans notre modèle économique construit sur des subventions venant du Nord et les actions envisagées dans leur pertinence locale. Ils souhaitent enfin tenter rompre plusieurs formes d'hégémonie dans la manière de penser et de mettre en œuvre les actions artistiques en Afrique. Organiser des non-événements, ou exprimer de manière positive des «moments », est pour Waza une invitation à considérer la création artistique du continent africain non pas dans ce qui fait l'événement, et qui, dans le contexte de l'attrait encore 
exotisant pour l'art africain se cantonnant au différent et au dissonant, permet de révéler beaucoup d'aspects, de luttes quotidiennes, de parcours sensibles dans une diffusion artistique qui devient une expérience de vie. Vu sous cet angle, tous les moments sont des bons moments pour découvrir le travail de Waza à Lubumbashi, parce qu'ils constituent autant de possibilité de "conversation» au sens étymologique de «vivre ensemble».

\section{References}

La publication Revolution Room est consultable sur le lien :

http://www.centredartwaza.org/wp-content/uploads/2017/08/Revolution-Room-Final-for-situ.pdf 